\title{
Impacto del Estilo Pedagógico Integrador en los Estudiantes de Licenciatura en Educación Básica de la Facultad De Estudios a Distancia
}

\author{
Claudia P. Moreno*, Yudy A. Molina y José A. Chacón \\ Universidad Pedagógica y Tecnológica de Colombia, Facultad de Estudios a Distancia, Sede Central \\ Tunja-Boyacá-Colombia, Avenida Central del Norte 39-115 \\ (e-mail: claudia.moreno@uptc.edu.co; yudy.molina@uptc.edu.co; jose.chacon@uptc.edu.co) \\ ${ }^{*}$ Autor a quien debe ser dirigida la correspondencia
}

Recibido Jun. 18, 2014; Aceptado Ago. 04, 2014; Versión final recibida Oct. 03, 2014

\begin{abstract}
Resumen
Se presenta un estudio y análisis sobre el estilo pedagógico integrador y su impacto en el aprendizaje de los estudiantes de Licenciatura en Educación Básica de la Universidad Pedagógica y Tecnológica de Colombia. Se describe la problemática sobre el conocimiento del estilo pedagógico real aplicado por los docentes de Licenciatura de la Facultad de Estudios a Distancia. El estudio es de carácter mixto no experimental, de tipo descriptivo y la muestra seleccionada fue de 250 estudiantes y 30 docentes. Los resultados revelan que los estilos de aprendizaje y enseñanza no son excluyentes entre sí, que los estudiantes tienden hacia el estilo de aprendizaje visual y kinestésico y se evidencia una gran preferencia por el estilo pedagógico integrador del docente. Esto demuestra que el educador universitario, formador de futuros profesores, debe reorientar su práctica para contribuir a la educación integral del ser, del saber y del saber hacer de los docentes en formación.
\end{abstract}

\section{Impact of Integrative Teaching Style on Students of the Basic Education Program of the Distance Studies Faculty}

\begin{abstract}
A study and analysis of the so-called integrative teaching style and its impact on learning of student belonging to the Basic Education Program of the Pedagogical and Technological University of Colombia are presented. The problems found on the knowledge of the real teaching style applied by teachers in courses of the Distance Studies Faculty are described. The study is of non-experimental mixed character and of descriptive type, and the selected sample was 250 students and 30 teachers. The results show that the learning styles and teaching methods are not mutually exclusive, students prefer visual and kinesthetic learning styles and there is evidence of the preference for the integrating style of the teacher. This demonstrates that the university educator, forming future teachers, must reorient his or her practice to contribute to the overall education of the person and to the knowledge and knowhow of the teacher being formed in our institutions.
\end{abstract}

Keywords: integrative teaching, teaching-learning, learning styles, teaching initiation 


\section{INTRODUCCIÓN}

En la sociedad del conocimiento del mundo globalizado en el que estamos inmersos, surgen retos en la educación como es el de mejorar la calidad de la misma; calidad que en buena parte está sujeta a los estilos de enseñanza que se apropian en los distintos ambientes de aprendizaje; puesto que es en el mundo de la escuela donde se ponen en juego interacciones entre los actores del proceso educativo (docente, estudiante). (Duarte, 2003). Es evidente que en el aula de clase se rompe el paradigma tradicional de enseñanza, abriendo oportunidades tanto para el docente como para el estudiante en la construcción del conocimiento bajo parámetros de interacción social. (Lobato y Madinabeitia, 2011). Teniendo en cuenta que el estilo pedagógico se enmarca en los roles que cumple el docente y el estudiante en el aula de clase, se pretende dar algunas aproximaciones conceptuales de este término.

Alonso (1994), define estilo a partir de un lenguaje pedagógico, señalándolo como una serie de distintos comportamientos asumidos acerca de la forma cómo actúan las personas, más allá de las apariencias. Y estilo de aprendizaje lo presenta como un indicador que guía las interacciones de la persona con la realidad existencial. Callejas (2005), señala que los estilos pedagógicos son "una manera característica de pensar el proceso educativo; una forma preferida de realizar la práctica y de poner en juego conocimientos, procedimientos, actitudes, sentimientos y valores”. Himmel (2001), presenta el estilo pedagógico, como la interrelación docente - estudiante que media el proceso educativo, a partir actitudes y comportamientos asumidos por los actores principales del acto educativo, reflejados en la praxis y en el perfeccionamiento de la misma. Para tal efecto, caracteriza la forma de enseñanza en cuatro categorías, así: estilo magistral, estilo planificador del proceso, estilo de pensamiento racional y crítico y estilo innovador e investigativo. Suárez et al. (2008), se fundamenta en los planteamientos expuestos por Himmel (2001) a partir de las concepciones de la interacción docente - estudiante, determinando cuatro estilos pedagógicos citados así: estilo directivo, estilo tutorial, estilo planificador y estilo investigativo:

Estilo directivo: es caracterizado por la disciplina, la severidad, la arbitrariedad y las clases magistrales; el maestro es el principal responsable del proceso; el alumno es pasivo y responde a los requerimientos del docente. Aquí predominan el contenido y la acción del maestro sobre el aprendizaje del alumno.

Estilo tutorial: aquí el maestro es un guía, un facilitador y mediador, que responde a los intereses y necesidades de los alumnos; el alumno, por su parte, es activo y autónomo. En este estilo se da un aprendizaje por descubrimiento, en el cual el proceso lo determina el alumno; hay actitud dialogante, el aprendizaje parte de las necesidades e intereses del alumno; se maneja una conciencia crítica.

Estilo planificador: el docente planea con anticipación sus actividades para apoyar a los alumnos, tomando en cuenta las diversas clases de aprendizaje e inteligencias, y es un evaluador en el proceso; el alumno responde y es activo. Aquí predomina la diversidad de estrategias, procurando un mayor aprendizaje de parte de los alumnos.

Estilo investigativo: es aquel que está orientado a la generación de nuevos problemas científicos, con una mirada compleja e interdisciplinaria; el alumno es activo, exigente y está en caminado hacia la producción científica. La solución de problemas científicos es la clave para desarrollo del conocimiento y del aprendizaje" (Suárez et al., 2008)

De acuerdo con los estilos anteriormente formulados, Moreno et al. (2010), proponen el estilo pedagógico integrador propio de los docentes de la Facultad de Estudios a Distancia, definiéndolo como: "El trabajo autónomo del estudiante a través de la utilización, implementación y aplicación de las herramientas de las tecnologías de la información y la comunicación. El estudiante dentro de un proceso de formación integral es responsable de su aprendizaje, teniendo en cuenta los valores de éste. Y el docente es mediador, guía y orientador del conocimiento, atendiendo a la flexibilidad del currículo con una mirada humanizadora. A la vez organiza, planifica con anticipación sus actividades y crea nuevas estrategias pedagógicas que surgen del desarrollo de la tecnología. De igual forma evalúa los procesos y contribuye a que el estudiante sea un constructor de nuevos conocimientos a partir de elementos innovadores, creativos e investigativos respondiendo a las necesidades de su contexto cultural y social" (Moreno et al, 2010)

La educación para el siglo XXI, exige educadores innovadores, creativos, investigativos e inquietos por los procesos de enseñanza aprendizaje, desde una realidad cambiante que genere estrategias y oportunidades para que los educandos asuman los desafíos de una manera responsable y autónoma, Recio (2010), El perfil del educador hoy en día, apunta a una enseñanza orientada a la producción lógica de conocimientos, desde una participación activa del estudiante invitándolo a discutir sus ideas y a compartir sus saberes, dejando de lado la monotonía y los procesos mecánicos y repetitivos que encasillan al estudiante en un rol tradicional (Rocha, 2013). Tarea no sencilla de lograr a partir del simple conocimiento que el docente posee 
sobre la disciplina objeto de enseñanza, sino a través de las actitudes y comportamientos asumidos por él, siendo un ente articulador de la ciencia, la tecnología y la educación desde una mirada problematizadora y contextualizada (Shulman, 2005)

El proyecto busca aportar al crecimiento del individuo a partir de la reflexión e investigación de los procesos que constituyen la academia, y las relaciones reciprocas e intrínsecas evidentes en los escenarios educativos a nivel de la modalidad en educación a distancia, convirtiéndose en una actividad humana que recae sobre el sujeto quien viene a ser el centro del proceso educativo brindando una educación flexible y autónoma que lo lleve al crecimiento evolutivo en todas sus dimensiones. (Altarejos, 1996). Makarenko (2001), propone el fortalecimiento de un estilo para trascender, formar valores colectivos y fomentar amor por el trabajo, una misión que acentúa esfuerzos por formar un hombre nuevo, reeducándolo, despertándole la autoestima a través de una pedagogía práctica. Desde esta perspectiva es importante crear espacios de reflexión a través de la praxis, la evaluación y la investigación rompiendo con el paradigma de una educación tradicional (Alder, 1991).

"La educación es ante todo una práctica social, que responde a, o lleva implícita una determinada visión del hombre (de lo que he llamado su crecer)" (Lucio, 1989). Freire (1970), sostiene que "el ser humano es un sujeto educable mientras vive". La actual visión de la educación para el nuevo siglo, implica cambios en el quehacer de los docentes y en su formación inicial y continua. Atendiendo a los retos de la sociedad del conocimiento a los que la educación invita a revisar con profundidad su esencia, en la cual el centro de la misma es el ser humano y su evolución, surge el interés por analizar el impacto de éste en el aprendizaje de los estudiantes de la Licenciatura en Educación Básica.

La problemática observada parte de la consolidación y de la determinación de un estilo propio de la Licenciatura, aunque se haya avanzado en su descubrimiento, se hace visible que no todos los docentes se identifican con el estilo pedagógico integrador, evidenciándolo en sus prácticas pedagógicas como en el desarrollo profesional de los estudiantes; a ello se suma, la falta de conocimiento de la metodología y del modelo pedagógico. En ocasiones los actores del proceso educativo, docentes y estudiantes operacionalizan sus prácticas haciéndolas poco productivas y llamativas en la construcción de nuevos conocimientos y de su propio aprendizaje. Cada maestro tiene un sello personal implícito y se quiere que éste sea reconocido a nivel universitario, para que su réplica favorezca los procesos educativos propuestos.

\section{MATERIALES Y METODOS}

El proyecto de investigación se enmarca en el enfoque mixto no experimental de tipo descriptivo, puesto que da cuenta de cómo suceden determinados fenómenos que caracterizan los estilos pedagógicos de los docentes y de qué manera influyen en el aprendizaje de los estudiantes. (McMillan y Schumacher, 2005). En la tabla 1, se describe la muestra seleccionada, la cual contó con la participación de 250 estudiantes matriculados de quinto a noveno semestre, población heterogénea compuesta por jóvenes entre 18 y 25 años, siendo en un gran porcentaje mujeres, provenientes de diferentes regiones del país y 30 docentes de la Licenciatura quienes participaron de forma voluntaria en el proceso.

Tabla 1: Muestra del estudio $(n=280)$

\begin{tabular}{|c|c|c|c|}
\hline Estudiantes & Mujeres & Hombres & Totales \\
\hline $\mathrm{V}$ & 45 & 5 & 50 \\
\hline $\mathrm{VI}$ & 42 & 1 & 43 \\
\hline $\mathrm{VII}$ & 38 & 4 & 42 \\
\hline $\mathrm{VIII}$ & 56 & 4 & 60 \\
\hline $\mathrm{IX}$ & 53 & 2 & 55 \\
\hline Docentes & 21 & 9 & 30 \\
\hline Totales & 255 & 25 & 280 \\
\hline
\end{tabular}

La variable principal seleccionada fue el estilo pedagógico integrador y la variable secundaria los estilos de aprendizaje de los estudiantes (Kinestésico-corporal, visual y auditivo). La metodología propuesta para el desarrollo del proyecto se centró en tres fases delimitadas de la siguiente manera: i) En la primera fase se aplicó una encuesta a estudiantes para identificar los diferentes estilos de aprendizaje, tomando como referencia el modelo de programación neurolingüística de Grinder (1989), también llamado visual-auditivokinestésico (VAK), el test contempla indicadores de cada uno de los estilos de aprendizaje. Reflejando, cómo el estilo pedagógico utilizado por el docente repercute en los aprendizajes de los estudiantes; ii) En la segunda fase, se diseñó y se aplicó una encuesta a estudiantes y entrevista a los docentes, teniendo en 
cuenta que las respuestas enriquecieran el acervo investigativo propuesto en este trabajo. La encuesta dirigida a los estudiantes se estructuró por medio de indicadores que caracterizan los distintos estilos pedagógicos de los docentes, como se muestra en la tabla 2, éstos se distribuyeron aleatoriamente en el formato para que el discente eligiera la opción que más se ajustara a la forma de enseñar de su docente, identificando de esta manera el estilo pedagógico del educador.

Tabla 2: Indicadores de los Estilos Pedagógicos del Docente (Adaptado de Suásres et al. (2008).

\begin{tabular}{|c|c|}
\hline Estilo Pedagógico & Indicadores \\
\hline Directivo & $\begin{array}{l}\text { a. Mantiene la disciplina, el orden y el silencio en clase. } \\
\text { b. Utiliza como estrategia de enseñanza la clase magistral } \\
\text { c. Le agrada que los estudiantes presten atención y tomen apuntes durante la clase. } \\
\text { d. Impone a sus estudiantes su forma de pensar. } \\
\text { e. Prescinde de espacios de participación y discusión que permitan la interacción docente - } \\
\text { f. Percibe en los estudiantes temor de preguntar y participar en clase. }\end{array}$ \\
\hline Planificador & $\begin{array}{l}\text { a. Entrega con anticipación la guía de aprendizaje a desarrollar durante el semestre. } \\
\text { b. Evalúa y realimenta permanentemente el proceso de aprendizaje } \\
\text { c. Entrega oportunamente el material de apoyo para el desarrollo de las actividades } \\
\text { académicas. } \\
\text { d. Da a conocer con antelación las formas de evaluación del proceso de aprendizaje. } \\
\text { e. Se apoya en diversas estrategias metodológicas para garantizar el éxito académico del } \\
\text { f. Induce al estudiante a planear y preparar con tiempo sus actividades académicas. }\end{array}$ \\
\hline Investigativo & $\begin{array}{l}\text { a. Centra la enseñanza en el enfoque investigativo e interdisciplinario. } \\
\text { b. Incentiva a los estudiantes a pensar de manera crítica frente a la evolución de la ciencia } \\
\text { c. Propone situaciones problémicas que lleven a los estudiantes a la búsqueda de soluciones } \\
\text { con una mirada reflexiva - activa. } \\
\text { d. Genera en sus estudiantes cuestionamientos sobre diversas problemáticas percibidas en su } \\
\text { contexto, invitándolos a buscar posibles soluciones. } \\
\text { e. Lleva al estudiante a aportar, crear, e innovar desde la teoría al contexto real. } \\
\text { f. Orienta al estudiante a visibilizar las posibles problemáticas reales, enfrentándolo a la } \\
\text { verificación metodológica de la misma. }\end{array}$ \\
\hline Tutorial & $\begin{array}{l}\text { a. Respeta los ritmos de aprendizaje de cada uno de los estudiantes. } \\
\text { b. Promueve el desarrollo de proyectos personalizados de trabajo. } \\
\text { c. Responde a los intereses y necesidades de los estudiantes. } \\
\text { d. Orienta, facilita y promueve el aprendizaje autónomo y colaborativo. } \\
\text { e. Acompaña permanentemente los procesos de aprendizaje, realimentando los conocimientos. } \\
\text { f. } \quad \text { Evalúa constantemente los procesos de perfeccionamiento y madurez del conocimiento. }\end{array}$ \\
\hline Integrador & $\begin{array}{l}\text { a. Utiliza las TIC como herramienta facilitadora del aprendizaje de sus estudiantes. } \\
\text { b. Propone estrategias pedagógicas derivadas de la evolución y desarrollo de las TIC. } \\
\text { c. Es un mediador, guía y orientador del conocimiento. } \\
\text { d. Atiende a la flexibilidad del currículo a través de los procesos investigativos. } \\
\text { e. Posee una mirada humanizadora desde el ser, el saber y el saber hacer. } \\
\text { f. Contribuye a que el estudiante sea un constructor de nuevos conocimientos a partir de } \\
\text { elementos innovadores, creativos e investigativos. }\end{array}$ \\
\hline
\end{tabular}

De los 250 estudiantes, 230 respondieron el test de estilos de aprendizaje y la encuesta sobre la percepción del estilo pedagógico del docente que corresponde a un (92\%). Los grupos de estudiantes se conformaron así: quinto semestre, $46(20 \%)$, sexto semestre, 39 (17\%), séptimo semestre $30(13 \%)$, octavo semestre, 60 (26.1\%) y noveno semestre, $55(23.9 \%)$. La mayoría de los estudiantes que participaron son docentes en ejercicio en diferentes regiones del país tanto en zonas rurales como urbanas (83.7\%), frente a un (16.3\%) que no tiene experiencia docente. El número total de docentes que participaron en la investigación es 30 , caracterizados por pertenecer a diferentes disciplinas de formación, en edades entre 30 y 50 años. Donde el $70 \%$ son mujeres y el $30 \%$ hombres.

También se diseñó una entrevista estructurada para los profesores con preguntas abiertas, con el fin de profundizar en la metodología utilizada tanto en los encuentros presenciales como en la asesoría ofrecida en el aula virtual a través de la plataforma Moodle. Se llevó a cabo la observación no participante de algunas sesiones de clase, registrando de manera puntual las acciones del acto educativo como soporte a la aplicación de los instrumentos, utilizando como categorías de análisis las características que identifican 
cada uno de los estilos pedagógicos, con el fin de contrastar la información suministrada en la aplicación de cada una de las técnicas para analizar el estilo pedagógico integrador y su impacto en el aprendizaje de los estudiantes como modelo y referente en la búsqueda de la calidad educativa en la Licenciatura en Educación Básica de la Facultad de Estudios a Distancia de la Universidad Pedagógica y Tecnológica de Colombia.

En la tercera fase, se determinó el impacto del estilo pedagógico integrador en el aprendizaje de los estudiantes mediante la triangulación de la información obtenida en cada una de las técnicas utilizadas, se retomaron los datos arrojados en la encuesta, entrevista y registro de observación para contrastarlos y hacer el análisis de contenido, destacando tres categorías como son: estilo pedagógico integrador, estilo de aprendizaje y actores del acto educativo (educador-educando).

\section{RESULTADOS Y DISCUSIÓN}

De acuerdo con la tabla 3, al comparar los estilos de aprendizaje de los estudiantes en cada uno de los semestres, se observa que los estilos que más predominan son el visual y el kinestésico, debido a que les facilita la apropiación del conocimiento puesto que aprenden haciendo, logrando así un aprendizaje significativo. De acuerdo con Romo et al. (2006), el modelo de estilos de aprendizaje de la Programación Neurolingüística (PNL) señala al criterio neurolingüístico, como la vía de ingreso de información al cerebro (ojo, oído, cuerpo) aspecto relevante en las particularidades de quién aprende o enseña. El sujeto posee tres métodos para organizar mentalmente la información: visual, auditivo y kinestésico.

Tabla 3: Estilos de aprendizaje de los estudiantes de la Licenciatura en Educación Básica

\begin{tabular}{|c|c|c|c|}
\cline { 2 - 4 } \multicolumn{1}{c|}{} & \multicolumn{3}{c|}{ Estilos de Aprendizaje } \\
\hline Semestre & Visual & Auditivo & Kinestésico \\
\hline V & 15 & 13 & 18 \\
\hline VI & 18 & 8 & 13 \\
\hline VII & 12 & 7 & 11 \\
\hline VIII & 20 & 15 & 25 \\
\hline IX & 19 & 12 & 24 \\
\hline Totales & 84 & 55 & 91 \\
\hline
\end{tabular}

A medida que los estudiantes avanzan en el proceso de formación académica, perciben en los docentes que el estilo pedagógico que los caracteriza es el Integrador (ver tabla 4), puesto que apropian elementos fundamentales de la modalidad de educación a distancia, asumiendo un rol de investigador, creativo, innovador y planificador, demostrando competencias en la evolución de las tecnologías de la información y la comunicación. "Un docente que muestre unos comportamientos de enseñanza moralmente admisibles y racionalmente fundamentados, no solamente enseñará la materia, sino que ayudara a los estudiantes a clarificar y controlar su razonamiento, a ser competentes en "aprender a aprender" (Renes et al., 2013)

Tabla 4. Estilos Pedagógicos percibidos por los Estudiantes de la Licenciatura en Educación Básica

\begin{tabular}{|c|c|c|c|c|c|}
\cline { 2 - 6 } \multicolumn{1}{c|}{} & \multicolumn{5}{c|}{ Estilos Pedagógicos } \\
\hline Semestre & Integrador & Investigativo & Planificador & Directivo & Tutorial \\
\hline V & 9 & 5 & 8 & 10 & 14 \\
\hline VI & 10 & 3 & 7 & 7 & 12 \\
\hline VII & 12 & 2 & 5 & 2 & 9 \\
\hline VIII & 21 & 3 & 11 & 9 & 16 \\
\hline$I X$ & 23 & 6 & 5 & 6 & 15 \\
\hline Totales & 75 & 19 & 36 & 34 & 66 \\
\hline
\end{tabular}

De la entrevista, se destacan las respuestas que los docentes dan a la pregunta: ¿qué metodología utiliza en el desarrollo de sus clases?, interrogante enfocado a caracterizar el estilo asumido por el docente en su praxis pedagógica. Algunas de las respuestas más relevantes son: 
Profesor 1: Estudio de casos, lectura atenta y concienzuda de la realidad, problematización de los hechos (realidad), análisis de la información para resaltar el perspectivismo de la verdad y las alternativas de solución, así mismo el análisis, la crítica y el juicio ponderado para dar sentido a lo real.

Profesor 2: Se utiliza una metodología activa y reflexiva enfocada a desarrollar las competencias en los estudiantes, se planifican los procesos de enseñanza aprendizaje atendiendo al enfoque y modelo propuesto por el programa de Licenciatura, se lleva a la práctica procesos de manera creativa e innovadora, evaluándolos desde un enfoque formativa propiciando un ambiente autónomo y colaborativo.

Profesor 3: Se da a conocer los criterios generales, se fijan compromisos y se establecen los grupos de trabajo. Los estudiantes realizan exposiciones donde se les cuestiona para aclarar dudas y complementar ideas.

En la tabla 5., se resumen los estilos que caracterizan a los 30 docentes del programa, se observa que los estilos que más predominan son el integrador con un $40 \%$ y el tutorial con un $30 \%$. Los docentes que asumen el estilo pedagógico integrador cuentan con una experiencia en el ejercicio de la docencia de más de 10 años, lo que les permite incorporar en su desempeño diferentes estrategias metodológicas propiciando ambientes favorables de aprendizaje.

Tabla 5. Estilos pedagógicos de los docentes de la Licenciatura en Educación

\begin{tabular}{|c|c|c|c|c|c|}
\hline & \multicolumn{5}{|c|}{ Estilos Pedagógicos } \\
\hline Docentes & Integrador & Investigativo & Planificador & Directivo & Tutorial \\
\hline Mujeres & 8 & 2 & 3 & 2 & 6 \\
\hline Hombres & 4 & & 1 & 1 & 3 \\
\hline Totales & 12 & 2 & 4 & 3 & 9 \\
\hline
\end{tabular}

Con base en la triangulación de la información se destacan tres categorías como son: estilo pedagógico integrador, estilo de aprendizaje y actores del acto educativo (educador - educando). En la figura 1, se puede observar el engranaje y articulación de éstas, donde el uso de los medios asincrónicos y sincrónicos son el punto en común que dinamizan la enseñanza y el aprendizaje dentro de una metodología flexible, reflexiva y sistemática. Se encuentra que hay coincidencias entre las percepciones que tienen los docentes de sí mismos con las percepciones que tienen los estudiantes.

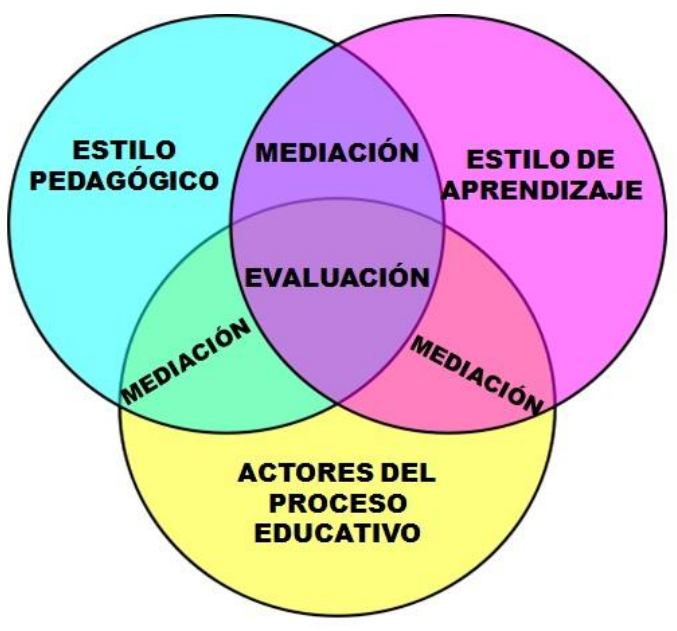

Fig.1 Triangulación: aprendizaje, estilo pedagógico y actores del proceso.

Desde una mirada de intersección matemática entre las tres categorías destacadas, la evaluación permite desde los distintos agentes un perfeccionamiento de la praxis a partir de la reflexión sobre cada uno de los eventos que se generan, como la autoevaluación, heteroevaluación, coevaluación y la misma metaevaluación. El proceso evaluativo lleva a un enriquecimiento personal y grupal tanto de los procesos académicos como de formación en los docentes y estudiantes, solidificando el modelo pedagógico desde la Educación a Distancia.

Se refleja el impacto del estilo pedagógico integrador del docente de la Facultad de Estudios a Distancia en el aprendizaje de los estudiantes de la Licenciatura en Educación Básica, determinando que la mediación se 
ha convertido en uno de los mayores desafíos en la educación, en razón a que los estilos pedagógicos y de aprendizaje contribuyen a la construcción de un modelo pedagógico propio, que atiende al crecimiento humano y perfeccionamiento de acciones dentro de un entorno real dando respuesta a los retos de la educación, la ciencia y la tecnología. Por otra parte, las herramientas de la información y la comunicación brindan oportunidades para diseñar ambientes de aprendizaje favoreciendo el trabajo colaborativo y cooperativo en la construcción de conocimiento en una comunidad de aprendizaje.

Es evidente, que tanto los estilos pedagógicos de los docentes como los estilos de aprendizaje de los estudiantes no son excluyentes entre sí, es decir, se complementan dada la interrelación que existe en cada uno de ellos con relación a la mediación estudiante - docente y viceversa. De esta manera, el docente deja permear la diversidad de opiniones y aportes con relación a las diferentes disciplinas y el estudiante presenta disponibilidad a un ambiente creativo, activo y diferente, proyectado al cambio para responder a lo imprevisto en su entorno social con el fin de lograr un ambiente adecuado en el aula y fuera de ella.

\section{CONCLUSIONES}

Del estudio realizado y los resultados presentados y analizados en este trabajo, se pueden obtener las siguientes conclusiones principales:

1. La interrelación existente en los procesos de enseñanza aprendizaje determina categorías tanto de estilos pedagógicos como de estilos de aprendizaje, que establecen claramente el rol del docente y del estudiante en los diferentes ambientes de aprendizaje.

2. Identificar los estilos de aprendizaje de los estudiantes ayuda al docente a generar un cambio en su praxis a través de la reflexión sobre la acción y a la búsqueda de nuevas metodologías, métodos y estrategias en la construcción de comunidades de aprendizaje.

3. La actividad docente se debe reorientar y reformular a la luz de los retos y desafíos que exige la evolución de la ciencia, la tecnología y la educación, bajo paradigmas de cambio en sus prácticas pedagógicas que contribuyan a la formación integral del ser, del saber y del saber hacer, respondiendo a una coherencia de vida desde su vocación, profesión y ocupación como integrantes de una sociedad.

\section{REFERENCIAS}

Alder, S. The reflective practitioner and the curriculum of teacher education. Journal of Education for teaching, 17(2) 139-150 (1991).

Alonso, C., Gallego, D., \& Honey, P.. Los estilos de aprendizaje. Procedimientos de diagnostico y mejora. Bilbao: Mensajero (1994)

Altarejos, F. Finalidad y libertad en educación. Anuario Filosófico, 29(2) 333-345 (1996).

Callejas, M. Universia. Los Estilos pedagógicos de los profesores universitarios. Recuperado el 15 de Marzo de 2012, de http://noticias.universia.net.co/en-portada/noticia/2005/12/21/257723/estilos-pedagogicosprofesores-universitarios.pdf (2005).

Duarte, J. Ambientes de Aprendizaje una aproximación conceptual. Estudios Pedagógicos, 29, 97-113 (2003)

Freire, P.. Pedagogía del Oprimido. Siglo XXI editores, México: (1970)

Grinder, J. B.. De sapos a príncipes. Editorial Cuatro Vientos, Chile (1989)

Himmel, E.. Evaluación del Aprendizaje en la Educación Superior. Calidad de la Educación, Consejo Superior de Educación de Chile, 79-89 (2001)

Lobato, C., y Madinabeitia, A. Perfiles Motivacionales del Profesorado ante la Formación en Metodologias Activas en la Universidad. Formación Universitaria, 4(1), 37- 48. (2011).

Lucio, R. Educación y pedagogía, enseñanza y didáctica: diferencias y relaciones. Revista de la Universidad de la Salle(17), 35-45 (1989)

Makarenko, A. Poema Pedagógico. Madrid, España: Akal (2001) 
McMillan, J., y Schumacher, S. Investigación Educativa (5 ed.). Madrid, España: Pearson (2005)

Moreno, C., Chacón, J., Aragón, J., Moreno, I., y González, W. Un estilo pedagógico para la educación a Ditancia. Prospectiva Científica(6), 130-137 (2010)

Recio, A. El perfil del educador para el siglo XXI. Publicaciones ICESI, 21-28 (2010)

Renes, P., Echeverry, L., Chiang, M., Rangel, L., y Martinez, P.. Estilos de enseñanza: un paso adelante en su conceptualización y diagnóstico. Revista Estilos de Aprendizaje, 11(11) 1-15 (2013)

Rocha, R.. Escala de Opinión de los Estudiantes sobre la Efectividad de la Docencia (EOEED) en Educación Superior. Formación Universitaria, 6(6), 13-22 (2013)

Romo, M., López, D., y López, I. ¿Eres visual, auditivo o kinestésico? Estilos de aprendizaje desde el modelo de la Programación Neurolingüística (PNL). Revista Iberoamerica de Educación, 38(2), 1-9 (2006).

Shulman, L. Conocimiento y enseñanza: fundamentos de la nueva reforma. Knowledge and Teaching: Foundations of the New Reform. Profesorado: Revista de curriculum y formación del profesorado, 9(2), 1-30 (2005)

Suárez, C.y otros 14 autores. Los estilos pedagógicos y su impacto en el aprendizaje de los alumnos (20012008). Bogotá D.C.: Fondo de Publicaciones Universidad Sergio Arboleda (2008) 